\title{
CISTO ODONTOGÊNICO GLANDULAR TRATAMENTO CIRÚRGICO POR MARSUPIALIZAÇÃO E ENUCLEAÇÃO: RELATO DE CASO
}

\author{
GLANDULAR ODONTOGENIC CYST SURGICAL TREATMENT BY \\ MARSUPIALIZATION AND ENUCLEATION: CASE REPORT
}

\author{
Letycia Maria Lopes de OLIVEIRA'; Gabriella Mendes OLIVEIRA'; Sergio Vitorino CARDOSO ${ }^{3}$; Flaviana Soares ROCHA ${ }^{4}$; \\ João César Guimarães HENRIQUES 5 \\ Residente em Oncologia do Programa de Residência Multiprofissional em Saúde Universidade Federal de Uberlândia. \\ Residente em Saúde Coletiva do Programa de Residência Multiprofissional em Saúde da Universidade Federal de Uberlândia. \\ Professor Associado da Área de Patologia da Faculdade de Odontologia da Universidade Federal de Uberlândia. \\ Professora Adjunta da Área de Cirurgia e Traumatologia Buco-Maxilo-Facial e Implantodontia da Faculdade de Odontologia da Universidade \\ Federal de Uberlândia. \\ Professor Adjunto I da Área de Diagnóstico Estomatológico, Faculdade de Odontologia, Universidade Federal de Uberlândia.
}

\section{RESUMO}

O cisto odontogênico glandular (COG) é o cisto de desenvolvimento mais agressivo e raro dentre todos os odontogênicos, portando características epiteliais glandulares ou salivares. $\mathrm{O}$ presente artigo visa retratar uma abordagem mais conservadora para esta importante lesão, no sentido de minimizar a morbidade decorrente da abordagem mais radical. Paciente com 57 anos, leucoderma, assintomático e edêntulo, compareceu ao ambulatório de estomatologia com notável expansão no fundo de saco vestibular e rebordo inferior direito, com tempo indeterminado de evolução. Os exames imaginológicos revelaram uma lesão radiolúcida unilocular extensa e bem delimitada na região anterior da mandíbula. Nas condutas propedêuticas, foi realizada uma punção aspiratória positiva para líquido cístico seguido de biópsia incisional. Diante destes achados, a marsupialização foi realizada no sentido de se conseguir a redução das dimensões lesionais, seguido da enucleação com estectomia periférica após 9 meses. Nos primeiros 2 anos de proservação o paciente mantém-se sem recidivas e em constante acompanhamento. Conclui-se que o dentista pode preferir o método conservador pela técnica de marsupialização seguida de enucleação na abordagem de extenso cisto odontogênico glandular, desde que haja colaboração do paciente nos cuidados pós-operatórios.

PALAVRAS-CHAVE: Cisto odontogênico glandular; Marsupialização; Patologia bucal.

\section{INTRODUÇÃO}

O cisto odontogênico glandular (COG) foi inicialmente descrito como cisto sialo-odontogênico por Padayache e Van Wyk ${ }^{1}$ (1987). Posteriormente, esta lesão foi reclassificada por Gardner et al. ${ }^{2}$ (1988) para cisto odontogênico glandular em virtude da sua derivação odontogênica. De acordo com a atual classificação dos tumores de cabeça e pescoço pela OMS, o COG é um cisto odontogênico de desenvolvimento com características epiteliais glandulares ou salivares ${ }^{3}$.

De acordo com a literatura, a frequência dos COGs variam 0,012 a $1,3 \%^{4}$, entre todos os cistos de origem odontogênica, sendo assim considerado um cisto raro, mas que merece atenção devido ao seu tamanho usualmente grande, ao seu aspecto multicístico e à elevada taxa de recidivas. É mais comumente diagnosticado na quinta década de vida, com uma ligeira predileção pelo sexo masculino. Cerca de $75 \%$ das lesões ocorrem na mandíbula e podem variar desde lesões pequenas de $1 \mathrm{~cm}$ assintomáticas até lesões maiores com sintomatologia ${ }^{5,6}$.

Radiograficamente, o COG exibe um padrão unilocular ou multilocular com bordos bem definidos e margens escleróticas. $\mathrm{O}$ aspecto histopatológico é variável, porém mais frequentemente se verifica revestimento epitelial estratificado de espessura variável. Caracteristicamente, as células superficiais são eosinofílicas, cubicas ou colunares, resultando em superfície irregular, por vezes papilar. Verifica-se eventualmente características apócrinas nas células superficiais, e com frequência, a presença de células caliciformes mucossecretoras e criptas intraepiteliais pseudocísticas, parcialmente preenchidas por material eosinofílico de aspecto semelhante a ductos, justificando a denominação desse cisto ${ }^{5,7,8}$.

Normalmente, as opções de tratamento incluem enucleação e curetagem, ressecção marginal, osteotomia periférica e ressecção parcial do maxilar (REF). Entretanto, estudos recentes têm mostrado que as grandes lesões também podem ser tratadas com marsupialização seguida de curetagem. Nesta modalidade de tratamento, em um primeiro momento é realizada a marsupialização, que consiste em uma cavidade cirúrgica na parede do cisto, permitindo a descompressão do seu conteúdo e mantendo a continuidade entre o cisto e a cavidade oral. Em um segundo momento, é realizada a exérese da lesão remanescente com curetagem ou ostectomia posterior ${ }^{9-11}$. 
O objetivo deste trabalho foi descrever um caso de COG, tratado pela modalidade conservadora da marsupialização seguida por enucleação e ostectomia periférica, evitando dessa forma morbidades maiores ao paciente decorrentes de terapias mais radicais como a ressecção.

\section{CASO CLÍNICO}

Paciente do gênero masculino, 57 anos, leucoderma, assintomático e edêntulo, compareceu ao ambulatório de estomatologia após seu implantodontista ter identificado uma lesão radiolúcida unilocular bem delimitada na mandíbula estendendo-se aproximadamente desde a região de mento, parassínfise e corpo mandibular direito (Figura 1A).

À oroscopia, foi observada mucosa íntegra, de coloração rósea e notável aumento volumétrico no fundo de saco vestibular e rebordo inferior direito, com tempo indeterminado de evolução (Figura 2). Na revisão da história médica, o paciente relatou acidente vascular cerebral há 5 anos, ser diabético, hipertenso e ex-fumante.

Para melhor delimitação e detalhamento da lesão, uma tomografia computadorizada de feixe cônico foi realizada identificando a hipodensidade da lesão bem delimitada, de aproximadamente $3,5 \mathrm{~cm}$ de diâmetro, que rompia corticais vestibulares e abrangia parte do canal mandibular (Figura 1B, 1C).

Inicialmente, foi proposto ao paciente a realização de punção aspirativa, seguida de biópsia incisional. Os níveis pressóricos do paciente apresentavam-se normais $(120 \mathrm{mmHg}$ x $80 \mathrm{mmHg})$ e a glicemia elevada $(200 \mathrm{mg} / \mathrm{dL})$ justificando antibioticoterapia com Amoxicilina 2 gramas previamente ao procedimento. Após aguardar 50 minutos, uma punção aspirativa foi realizada revelando líquido sanguinolento e amarelado. Em seguida, sob anestesia local, foi removida parte da cápsula lesional, que foi fixada em formalina e encaminhada para análise anátomo-histopatológica. $\mathrm{O}$ remanescente da cápsula foi exposto e suturado conjuntamente com a mucosa adjacente, caracterizando a técnica da marsupialização.

O laudo histopatológico mostrou fragmentos teciduais de cápsula cística parcialmente revestida por tecido epitelial. A parede de tecido conjuntivo apresentou-se constituída por tecido denso, não modelado, permeado por focos de infiltrado inflamatório mononuclear. O tecido epitelial apresentou-se em parte estratificado, cúbico e colunar, ciliado, em parte mostrando-se pavimentoso estratificado. Focalmente, foram observadas estruturas ductóides na espessura do epitélio, e eventuais focos de espessamento com enovelamento das células epiteliais, diagnosticado como cisto odontogênico ontogênico glandular (Figura 3 - Corte histopatológico).

O paciente foi orientado a realizar irrigações diárias com digluconato de clorexidina $0,12 \%$ no interior da lesão após as refeições e foi proservado clinicamente todos os meses e imaginologicamente a cada 3 meses. Após 9 meses, uma neoformação óssea satisfatória foi verificada e o paciente submetido à enucleação final com posterior ostectomia periférica (Figura 4). Foi realizada uma tomografia 12 meses após a enucleação, que evidenciou remodelação óssea da região (Figura 5). O paciente encontra-se em contínua proservação, com dois anos de acompanhamento, encontra-se bem e sem qualquer indício de recidivas (Figura 6).

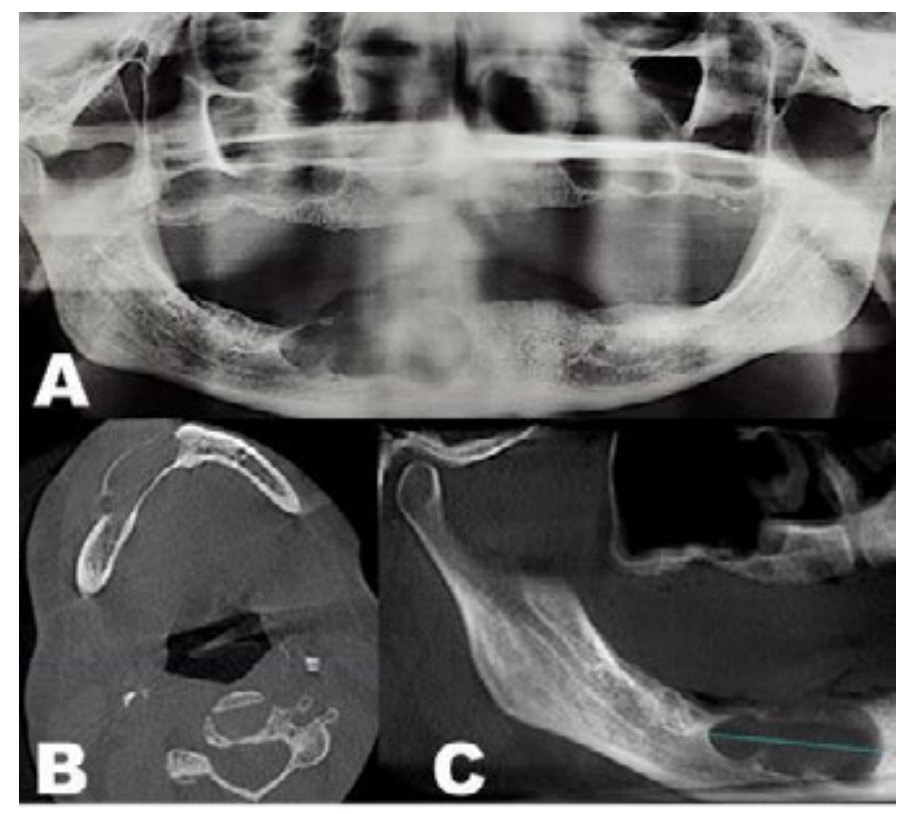

Figura 1(A) - Radiografia panorâmica inicial. (B) - Corte axial de tomografia computadorizada apresentando descontinuidade óssea em região vestibular mandibular lado direito. (C) - Corte panorâmico de tomografia computadorizada identificando a hipodensidade da lesão, de aproximadamente $3,5 \mathrm{~cm}$ de diâmetro, rompendo corticais vestibulares e abrangendo parte do canal mandibular.

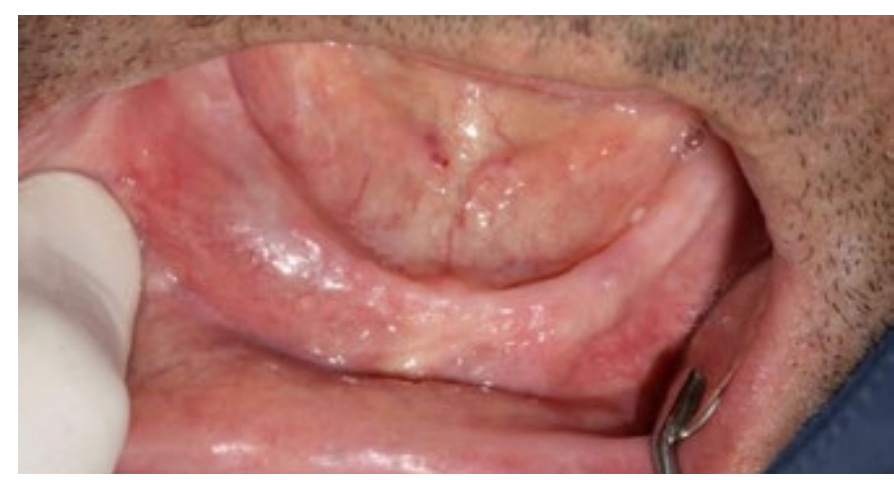

Figura 2 - Aspecto inicial do caso clínico, elucidando notável aumento volumétrico no fundo de saco vestibular e rebordo inferior direito.

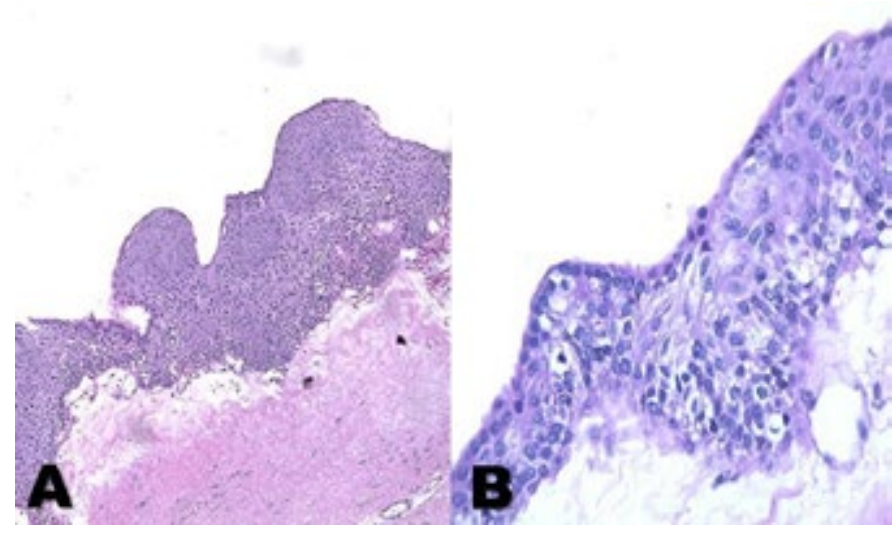

Figura 3 - Corte histopatológico. Presença de fragmentos teciduais de cápsula cística parcialmente revestida por tecido epitelial. A parede de tecido conjuntivo apresentou-se constituída por tecido denso, não modelado, permeado por focos de infiltrado inflamatório mononuclear. $\mathrm{O}$ tecido epitelial apresentou-se em parte estratificado, cúbico e colunar, ciliado, em parte mostrando-se pavimentoso estratificado. Focalmente, foram observadas estruturas ductóides na espessura do epitélio, e eventuais focos de espessamento com enovelamento das células epiteliais. 


\section{DISCUSSÃO}

O cisto odontogênico glandular é um cisto de desenvolvimento raro cujo tratamento é atualmente digno de muitos estudos e controvérsias na literatura mundial. De forma geral, as modalidades terapêuticas oscilam de acordo com a dimensão da lesão, sendo que lesões menores e uniloculares seriam mais bem tratadas por enucleação e ostectomia periférica e lesões maiores e multiloculares seriam mais bem conduzidas por ressecção marginal. Há ainda na literatura a opção pela marsupialização das lesões de maiores dimensões, especialmente considerando o envolvimento de estruturas nobres próximas ao campo operatório $^{12,13}$.

A marsupialização é um procedimento cirúrgico conservador frequentemente utilizado em diversas lesões dos maxilares, tais como queratocistos, cistos dentígeros, cistos do ducto nasopalatino, ameloblastomas unicísticos e cistos epiteliais calcificantes, que permite a descompressão cística e consequente regressão da lesão, determinando, na maioria das vezes, um ótimo prognóstico e preservação dos tecidos ao paciente ${ }^{14}$.

Estudos têm mostrado que a técnica de marsupialização seguida de enucleação apresentam menor morbidade ao paciente e também à preservação de estruturas anatômicas nobres ${ }^{11}$. A lesão do caso apresentado, mostrava uma associação com parte do canal mandibular e consequente inervação envolvida, de modo que uma ressecção cirúrgica implicaria além de grande perda tecidual na região, a parestesia permanente do paciente, implicando assim em grande morbidade.

Chrcanovic e Gomez ${ }^{14}$ (2017) ao analisarem 169 casos de COG, constaram uma taxa de recidiva de $21,6 \%$. Afirmaram que procedimentos adjuvantes após a enucleação do cisto devem ser necessariamente considerados, destacando, por exemplo, a crioterapia, solução de Carnoy ou ostectomia periférica ${ }^{14,15}$. Neste estudo não houve terapias adjuvantes além da ostectomia periférica realizada, muito embora pudesse ser de interesse associá-las após a curetagem ou ostectomia finais ${ }^{14}$.

Finalmente, vale destacar a importância de se esclarecer ao paciente a necessidade absoluta do acompanhamento periódico por um período mínimo de 7 anos, uma vez que o potencial de recidiva destas lesões é inegavelmente alto ${ }^{13}$.

\section{CONCLUSÃO}

O presente caso relatou um cisto odontogênico glandular em mandíbula e o desafio na condução terapêutica. Têm por objetivo final destacar a importância de sempre considerar terapias mais conservadoras na abordagem das lesões dos maxilares, quando houver suporte da literatura mundial. Embora possa haver maior propensão às recidivas, quando comparado a terapias mais radicais como a ressecção marginal, tratamentos como a marsupialização seguida de enucleação e curetagem ou ostectomia, podem ter excelente prognóstico se bem conduzidos, além de permitir considerável melhora na qualidade de vida dos pacientes quando comparado às modalidades menos conservadoras.

\section{REFERÊNCIAS}

01. Padayachee A, Van WYC. Two cystic lesions with features of both the botryoid odontogenic cyst and the central mucoepidermoid tumour: sialo-odontogenic cyst? J Oral Pathol.1987;16(10):499-04.

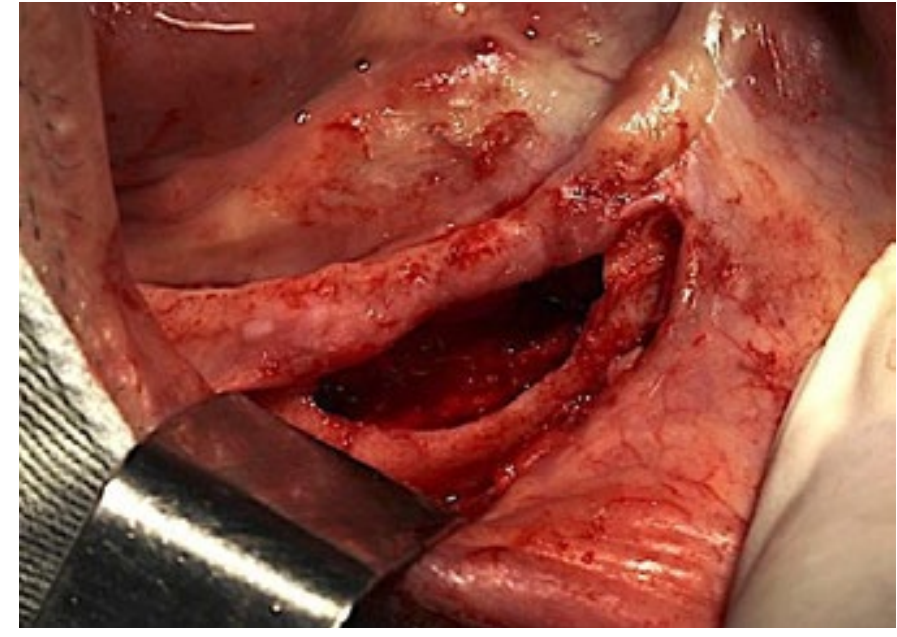

Figura 4 - Enucleação da lesão com ostectomia periférica.

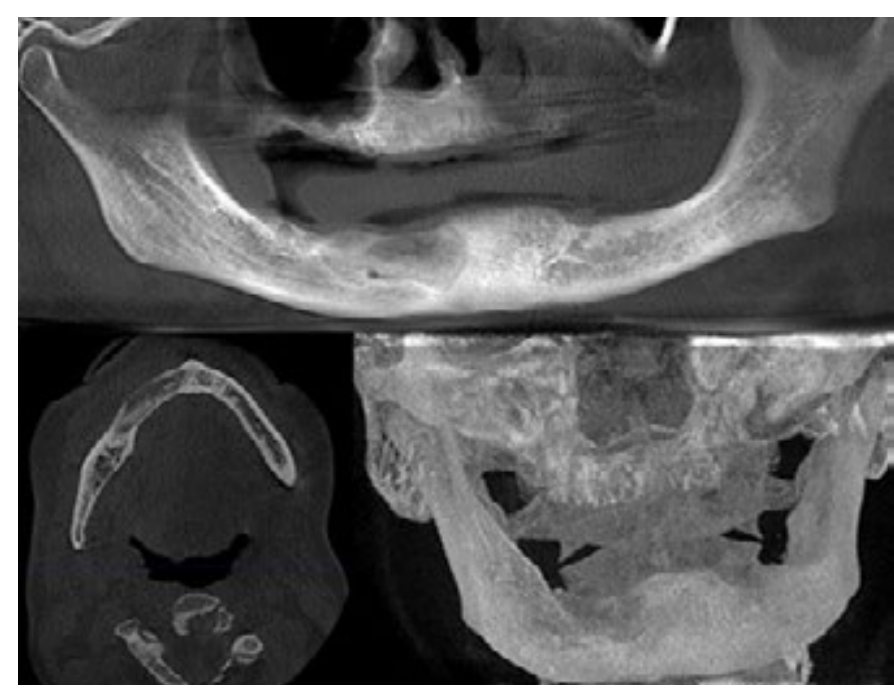

Figura 5 - Acompanhamento tomográfico 1 ano após enucleação, apresentando neoformação óssea satisfatória.

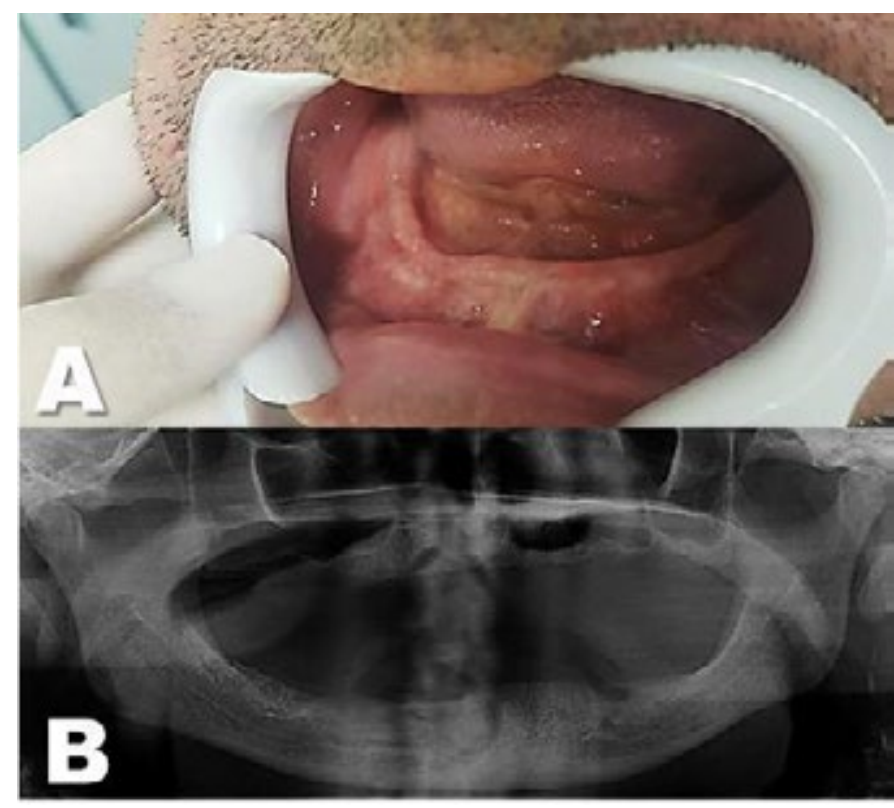

Figura 6 (A) - Aspecto final do caso clínico, após 2 anos de acompanhamento. (B) Radiografia panorâmica após 2 anos de acompanhamento, sem sinais de recidiva. 
02. Gardner DG, Kessler HP, Morency R, Schaffner DL. The glandular odontogenic cyst: An apparent entity. Journal of Oral Pathology. 1988; 17(8):359-66.

03. El-Naggar AK, Chan JKC, Grandis JR, Takata T, Slootweg PJ. World Health Organization Classification of Head and Neck Tumours. 4.ed. Lyon, France: WHO; 2017.

04. Manzini H, Deon C, Corte LD, Bertotto JC, Abreu LB. Odontogênico glandular cisto: uma entidade incomum. Braz J Otorhinolaryngol. 2009; 75(2):320.

05. Neville BW, Damm DD, Allen CM, Bouquot JE. Oral and Maxillofacial Pathology. 3.ed. New York: WB Saunders CO; 2009.

06. Mittal A, Narang V, Kaur G, \& Sood N. Glandular Odontogenic Cyst of Mandible: A Rare Entity. Journal of Clinical and Diagnostic Research. 2015: 9(12):09-1.

07. Fowler CB, Brannon RB, Kessler HP, Castle JT, Kahn MA. Glandular odontogenic cyst: analysis of 46 cases with special emphasis on microscopic criteria for diagnosis. Head Neck Pathol. 2011; 5(4)364-75.

08. Shear M, Speight P. Glandular odontogenic cyst. IN: Cysts of the oral and maxillofacial regions. 4. ed. Oxford: Blackwell-Munksgaard; 2007.
09. Kaplan I, Gal G, Anavi Y, Manor R, Calderon S. Glandular odontogenic cyst: treatment and recurrence. J Oral Maxillofac Surg. 2005; 63(4):435-41.

10. Oliveira NJP, Avelar RL, Andrade ESS, Raimundo RC, Gomes ACA, Laureano FJR. Tratamento cirúrgico de extenso cisto odontogênico glandular: considerações clínico-cirúrgicas. Rev Cir Traumatol Bucomaxilo-fac. 2009; 9(4):59-5.

11. Pinto GNS, Figueira JA, Gonçales ES, Sant'ana E, Tolentino ES. Marsupialização como tratamento definitivo de cistos odontogênicos: relato de dois casos. RFO UPF. 2015; 20(3):361-66.

12. Shah M, Kale H, Ranginwala A, Patel G. Glandular odontogenic cyst: A rare entity. J Oral Maxillofac Pathol. 2014; 18(1):89-2.

13.Zanon C, Cavalcanti MA, Hespanhol W. Cisto odontogênico glandular: uma condição patológica incomum: revisão de literatura. Clipe Odonto. 2016; 8(1):38-3.

14. Chrcanovic BR, Gomez RS. Glandular odontogenic cyst: An updated analysis of 169 cases reported in the literature. Oral Dis. 2018; 24(5):717-24.

15. Anchlia S, Bahl S, Shah V, Vyas S. Glandular odontogenic cyst: A rare entity revealed and a review of the literature. BMJ Case Rep. 2015; $5: 45-1$.

\section{ABSTRACT}

The glandular odontogenic cyst (GOC) is the most aggressive and rare cyst of development among all odontogenics, with glandular or salivary epithelial characteristics. This article aims to portray a more conservative approach to this important lesion, in order to minimize the morbidity resulting from the more radical approach. Patient 57 years-old, leucoderma, asymptomatic and edentulous, attended the stomatology outpatient clinic with a remarkable expansion in the vestibular bag fundus and lower right border, with indeterminate evolution time. Imaging examinations revealed an extensive and well delimited unilocular radiolucent lesion in the anterior region of the mandible. In the propaedeutic procedures, a positive aspiration puncture was performed for cystic fluid followed by incisional biopsy. In view of these findings, marsupialization was performed in order to reduce lesion dimensions, followed by enucleation with peripheral ostectomy after 9 months. In the first 2 years of proservation, the patient remains without relapses and in constant follow-up. It is concluded that the dentist may prefer the conservative method by the marsupialization technique followed by enucleation in the approach of extensive glandular odontogenic cyst, provided there is collaboration of the patient in the postoperative care.

KEYWORDS: Glandular odontogenic cyst; Marsupialization; Pathology oral.

\section{AUTOR PARA CORRESPONDÊNCIA}

Letycia Maria Lopes de Oliveira

Universidade Federal de Uberlândia - Avenida Pará, 1720,

bloco 4T, Campus Umuarama, CEP 38405-900.

Telefone: +55(34)998220773

E-mail: letycialopes257@gmail.com 